\title{
Transcriptome analysis of flavonoid biosynthesis in safflower flowers grown under different light intensities
}

\author{
Chaoxiang Ren ${ }^{1,2}$, Jie Wang ${ }^{1,2}$, Bin Xian ${ }^{1,2}$, Xiaohui Tang ${ }^{1,2}$, Xuyun Liu ${ }^{3}$, Xueli Hu ${ }^{3}$, Zunhong Hu ${ }^{3}$, Yiyun Wu ${ }^{1,2}$, \\ Cuiping Chen ${ }^{1,2}$, Qinghua Wu ${ }^{1,2}$, Jiang Chen ${ }^{\text {Corresp., 1, } 2}$, Jin Pei ${ }^{\text {Corresp. 1, } 2}$ \\ ${ }^{1}$ College of Pharmacy, Chengdu University of Traditional Chinese Medicine, Chengdu, China \\ 2 Development and Utilization of Chinese Medicine Resources, State Key Laboratory Breeding Base of Systematic Research, Chengdu, China \\ 3 Industrial Crop Institute of Yunnan Academy of Agricultural Sciences, Yunnan, China \\ Corresponding Authors: Jiang Chen, Jin Pei \\ Email address: janshen1986@163.com, peixjin@163.com
}

Background. Safflower (Carthamus tinctorius L.) is a domesticated species with a long history of cultivation and widespread distribution across the globe, and light plays an important role in controlling its distribution boundary. Flowers from safflower have been widely used in traditional Chinese medicine because of their ability to improve cerebral blood flow. Flavonoids are the main active compounds in safflower and have many pharmacological effects. In this study, we aimed to explore the relationship between different light intensities and flavonoid biosynthesis in safflower flowers cultivated in greenhouse.

Methods. The transcriptome of safflower flowers grown under different light intensities were sequenced through BGISEQ-500 platform. After assembled and filtered, Unigenes were annotated by aligning with 7 functional databases. Differential expression analysis of two samples was performed with the DEseq2 package. Differentially expressed genes (DEGs) related with flavonoids biosynthesis were analyzed by Quantitative Real-time PCR (RT-PCR). Flavonoids accumulation in flowers were determined by high performance liquid chromatography (HPLC) and spectrophotometer.

Results. Transcriptome analysis of safflower flowers cultivated under different light intensities was performed. A total of 99.16 GB data were obtained, and 78,179 Unigenes were annotated. Among the DEGs, 13 genes were related to flavonoid biosynthesis. The differential expressions of 7 key genes were confirmed by RT-PCR. In addition, the levels of some flavonoids were measured in safflower flowers grown under different light intensities. CHHCT3 gene expression showed a significantly negative correlation with kaempferol content in safflower grown under different light intensities.

Conclusion. Our results strongly suggested that the reduction in light intensity in a suitable range promoted flavonoid biosynthesis in safflower flowers. We suggest that the expression of HCT genes played an important role in flavonoid accumulation in safflower flowers. Our study lays a foundation for further research on the effects of light on flavonoid biosynthesis in safflower. 
1 Transcriptome analysis of flavonoid biosynthesis in safflower flowers

2 grown under different light intensities

3 Chaoxiang Ren ${ }^{1,2}$, Jie Wang ${ }^{1,2}$, Bin Xian ${ }^{1,2}$, Xiaohui Tang ${ }^{1,2}$, Xuyun Liu ${ }^{3}$, Xueli Hu ${ }^{3}$, Zunhong Hu ${ }^{3}$,

4 Yiyun Wu ${ }^{1,2}$, Cuiping Chen 1, , , Qinghua Wu ${ }^{1,2}$, Jiang Chen Corresp.., 1, 2, Jin Pei Corresp. 1, 2

$5 \quad{ }^{1}$ College of Pharmacy, Chengdu University of Traditional Chinese Medicine, Chengdu 611137, Sichuan, China

$6 \quad{ }^{2}$ State Key Laboratory Breeding Base of Systematic Research, Development and Utilization of Chinese Medicine

7 Resources, Chengdu 611137, Sichuan, China

$8 \quad{ }^{3}$ Industrial Crop Institute of Yunnan Academy of Agricultural Sciences, Kunming 650205, Yunnan, China

9 Corresponding Authors: Jiang Chen, Jin Pei

10 Email address: janshen1986@163.com, peixjin@163.com

\section{Abstract}

Background. Safflower (Carthamus tinctorius L.) is a domesticated species with a long history of cultivation and widespread distribution across the globe, and light plays an important role in controlling its distribution boundary. Flowers from safflower have been widely used in traditional Chinese medicine because of their ability to improve cerebral blood flow. Flavonoids are the main active compounds in safflower and have many pharmacological effects. In this study, we aimed to explore the relationship between different light intensities and flavonoid biosynthesis in safflower flowers cultivated in greenhouse.

Methods. The transcriptome of safflower flowers grown under different light intensities were sequenced through BGISEQ-500 platform. After assembled and filtered, Unigenes were annotated by aligning with 7 functional databases. Differential expression analysis of two samples was performed with the DEseq2 package. Differentially expressed genes (DEGs) related with flavonoids biosynthesis were analyzed by Quantitative Real-time PCR (RT-PCR). Flavonoids accumulation in flowers were determined by high performance liquid chromatography (HPLC) and spectrophotometer.

Results. Transcriptome analysis of safflower flowers cultivated under different light intensities was performed. A total of $99.16 \mathrm{~GB}$ data were obtained, and 78,179 Unigenes were annotated. Among the DEGs, 13 genes were related to flavonoid biosynthesis. The differential expressions of 7 key genes were confirmed by RT-PCR. In addition, the levels of some flavonoids were 
measured in safflower flowers grown under different light intensities. CtHCT3 gene expression showed a significantly negative correlation with kaempferol content in safflower grown under different light intensities.

Conclusion. Our results strongly suggested that the reduction in light intensity in a suitable range promoted flavonoid biosynthesis in safflower flowers. We suggest that the expression of $H C T$ genes played an important role in flavonoid accumulation in safflower flowers. Our study lays a foundation for further research on the effects of light on flavonoid biosynthesis in safflower.

\section{Introduction}

Safflower, Carthamus tinctorius L. $(2 \mathrm{n}=2 \mathrm{x}=24)$, is a member of the family Asteraceae. It is an annual and a predominantly self-pollinated species. Safflower is a domesticated species with a long history of cultivation and widespread distribution across the world (Dempewolf, Rieseberg \& Cronk, 2008). With the introduction of inexpensive synthetic dyes in the middle of the last century, the importance of its flowers as a source of dye has almost vanished. However, the breeding of safflower for its high oil content and the modified fatty acid composition in its seeds (Knowles, 1972) has given rise to new varieties that led to its re-establishment as an important high-quality oil crop for cooking and industrial purposes in many parts of the world (Ashri et al., 1977). On the basis of the morphological variability that exists in Carthamus tinctorius L., researchers proposed seven 'centers of similarity' (the Far East, India-Pakistan, the Middle East, Egypt, Sudan, Ethiopia, and Europe) that have predominant morphotypes at each center (Knowles, 1969). However, China, unlike other countries, has been using its flowers as a medicinal herb for nearly 2000 years and has already become a special cultivation center. Previous studies on the effect of environmental factors on safflower distribution, showed that light was the most important factor explaining safflower distribution boundaries $(\mathrm{Wu}, 2017)$. Plants have adopted the ability to sense light signals which is one of the most important environmental factors for plants growth, including light quantity (intensity), quality (wavelength), direction and duration. The response of plants to light occurs in various developmental processes, such as seedling photomorphogenesis, circadian rhythms, flower induction as well as the accumulation of secondary metabolites (Jiao, Lau \& Deng, 2007). Numerous studies have shown that light intensity can influence the biosynthesis of flavonoids in other species. For instance, shade had a significantly negative effect on contents of total flavonoid, kaempferol, quercetin, and isoquercitrin in leaves of Cyclocarya paliurus. However, the greatest accumulation of total flavonoid in the leaves was observed in intermediate shade treatment (Deng et al., 2012). In Epimedium pseudowushanense B.L.Guo, the flavonoid contents varied with 5 different light intensity levels (light intensity was getting stronger from level 1 to 
level 5). And the largest amount of epimedin $\mathrm{C}$ was produced at light intensity level 4 (Pan et al., 2017). In Begonia semperflorens, high light stress promoted anthocyanin synthesis in the leaves (Wang et al., 2018).

Flavonoids are ubiquitous secondary metabolites that have various functions in plant physiology and ecology (Tian, Pang \& Dixon, 2008). At present, the basic metabolic pathway of flavonoid biosynthesis is well known, especially in Arabidopsis thaliana (Hai, Huang \& Tang, 2010; Saito et al., 2013). P-coumaroyl CoA is the substrate of two enzymes at the junction of the metabolic routes leading to flavonoids or to phenylpropanoid compounds (Fig. 1). One of enzymes is shikimate O-hydroxycinnamoyl transferase (HCT) [EC:2.3.1.133], which leads to biosynthesis of two major lignin building units, namely, the guaiacyl and syringyl units (Besseau et al., 2007). Another is chalcone synthase (CHS), which catalyzes $p$-coumaroyl CoA and three malonyl CoA molecules to form the naringenin chalcone, and naringenin chalcone subsequently is converted to naringenin by chalcone isomerase (CHI). Flavanone 3-hydroxylase $(\mathrm{F} 3 \mathrm{H})$, flavonol synthase (FLS) [EC:1.14.20.6] and (F3'H) catalyze the formation of many kinds of flavonol, such as kaempferol and quercetin. Downstream, anthocyanidin synthase (ANS) [EC:1.14.20.4] catalyzes the formation of anthocyanin, such as pelargonidin and cyanidin. However, only a few genes have been identified in flavonoid biosynthesis pathway of safflower, such as $\mathrm{CHI}$ and $\mathrm{F3H}$ (Guo et al., 2019; Ren et al., 2019; Tu et al., 2016). Therefore, transcriptome analysis is one of the best approaches to find functional genes and to reveal the flavonoid biosynthesis in safflower. Additionally, pharmacological studies have shown that flavonoids in safflower have several pharmacological effects including cardioprotective (Jin et al., 2008), vasodilative effects and exhibit anti-hypertensive (Asgarpanah \& Kazemivash, 2013), anti-coagulation and antithrombotic (Hong et al., 2015) properties. Specially, hydroxysafflor yellow A (HSYA) is the most widely studied chemical in safflower. Recent research reports showed that HSYA had antiinflammatory activity and a protective effect on ischaemic cardiac dysfunction, and it was able to weaken coronary heart disease and enhance the rate of wound closure (Gao et al., 2018; Zhou et al., 2018; Zou et al., 2018). Besides, kaempferol is a natural flavonol present in different plant species that possesses potent anti-inflammatory properties (Devi et al., 2015). Kaempferol, as an index compound of safflower, is listed along with HSYA in the Chinese Pharmacopoeia (Chinese Pharmacopoeia Commission, 2015).

To reveal the relationship between light intensity and flavonoid biosynthesis in safflower, transcriptomes of safflower flowers grown under different light intensities were sequenced in this study. Different genes related to flavonoid biosynthesis were analyzed. In addition, HSYA, kaempferol and total flavonoid contents in the safflower flowers were determined. The correlation between gene expression and the flavonoid content was analyzed. Our results provide a basis for further study of the influence of light intensity on flavonoid biosynthesis in safflower 
flowers.

\section{Materials and Methods}

\section{Plant materials}

The safflower flowers used in this study were obtained from the phytotron at the Chengdu University of Traditional Chinese Medicine in Chengdu, China. Prior to the experiment, the plants were grown at $25^{\circ} \mathrm{C}$ during the day and at $18^{\circ} \mathrm{C}$ at night; the relative humidity ranged from $50 \%$ to $60 \%$. Safflower plants with the same growth status were selected and grown in a peat: vermiculite: perlite $(3: 1: 1)$ mixture in a phytotron room. The 8 months old plants were divided into three groups (30 independent samples in each group) with equal size buds. Plants were illuminated with light-emitting diodes (LEDs) among three groups. The high light (HL) group was grown under 40,000 Lux. The second group, middle light (ML), was grown under 20,000 Lux. The third group, low light (LL), was grown under 10,000 Lux. Light intensity settings referred to light compensation point (LCP) and light saturation point (LSP) of safflower (Guan et al., 2007). Three different treatments lasted from budding to blooming. Blooming flowers were collected per group from the same location on the plants. These samples were immediately frozen at $-80^{\circ} \mathrm{C}$. Three biological replicates were used in each treatment.

\section{RNA extraction}

Flowers were ground in liquid nitrogen, and the total RNA was isolated by TRIzol reagent (Invitrogen, CA, USA). To remove DNA, an aliquot of total RNA was treated with DNase (Takara, Dalian, China) by using the standard protocol described by the manufacturer. The purity of the RNA samples according to the A260/A280 ratio was determined with a NanoDrop; the A260/A280 ratios of all samples were in the approximate range of 1.9-2.1. The integrity of the RNA samples was assessed with an Agilent 2100 Bioanalyzer, and samples with no sign of degradation were selected for further analysis.

\section{Library construction and sequencing}

A total of 9 samples, 3 per treatment, were randomly selected to constructed libraries. The first step in the workflow involved purifying the poly-A containing mRNA molecules using poly-T oligo-attached magnetic beads. Following purification, the mRNA was fragmented into small pieces through divalent cations under elevated temperature. The cleaved RNA fragments were copied into first strand cDNA using reverse transcriptase and random primers. This was followed by second strand cDNA synthesis employing DNA Polymerase I and RNase H. A single 'A' base was then added to these cDNA fragments, and an adapter was subsequently ligated to the cDNA fragments. The products were then purified and enriched with PCR amplification. Then, the PCR yield was quantified by Qubit, and samples were pooled together to make a singlestrand circular DNA (ssDNA circle), which was the final library. DNA nanoballs (DNBs) were 
137 generated with the circular ssDNA by rolling circle replication (RCR) to enlarge the fluorescent

138 signals during the sequencing process. The DNBs were loaded into the patterned nanoarrays, and 139 pair-end reads of $100 \mathrm{bp}$ were read with the BGISEQ-500 platform for the following data 140 analysis. For this step, the BGISEQ-500 platform combined the DNA nanoball-based nanoarrays and stepwise sequencing employing the combinational probe-anchor synthesis sequencing method. Raw reads containing a low quality, adaptor-polluted and a high content of unknown bases $(\mathrm{N})$ were removed before downstream analyses. Reads cleaning and filtering were performed by BGI internal software.

\section{De novo assembly and functional annotation}

Because published genome of safflower was not integrated, this project was done without a reference genome. Clean reads in each sample were assembled independently to obtain a reference sequence for subsequent analysis. De novo transcriptome assembly was completed by Trinity v2.0.6 (Grabherr et al., 2011) with clean reads, and then TGICL v2.0.6 (Pertea et al., 2003) was used on cluster transcripts to remove redundant transcripts and obtain Unigenes. The Unigenes were divided into two types: one type was a cluster in which the prefix was CL with the cluster ID behind it. In one cluster, there are several Unigenes which similarity between them is more than $70 \%$. Another type was a singleton in which the prefix was Unigene. The N50, N70 and N90 length were used to determine the assembly continuity. The higher the length of N50, N70 and N90, the better the assembling continuity was. The raw data were uploaded to the NCBI and Figshare.

Unigene sequences were aligned to the following databases with BLAST (v2.2.23): NT (ftp://ftp.ncbi.nlm.nih.gov/blast/db), NR (ftp://ftp.ncbi.nlm.nih.gov/blast/db), KOG (http://www.ncbi.nlm.nih.gov/KOG), KEGG (http://www.genome.jp/kegg) and SwissProt (http://ftp.ebi.ac.uk/pub/databases/swissprot). Based on the NR annotation, GO functional annotation (http://geneontology.org) was obtained through the Blast2GO program (v2.5.0). InterPro annotations (http://www.ebi.ac.uk/interpro) was obtained by InterProScan 5.

\section{Analysis of the DEGs}

Clean reads were mapped to the Unigenes by Bowtie2 v2.2.5 (Langmead \& Salzberg, 2012), and then the gene expression levels were calculated through RSEM v1.2.12 ( $L i \&$ Dewey, 2011). Differential expression analysis was performed by DEseq2 as described by Love et al. (Love, Huber \& Anders, 2014). DEseq2 is a package implemented in $\mathrm{R}$ based on the negative binomial distribution. A fold change $\geq 2.00$ and an adjusted $P$ value $\leq 0.05$ were set as the thresholds to establish significantly differential expression. Classification and functional enrichment analysis including Gene Ontology (GO) and KEGG were performed to identify which GO terms or metabolic pathways were significantly enriched in the DEGs.

\section{RT-PCR expression analysis of DEGs}


173 Because of the pharmacological effects of safflower flavonoid, we focused on DEGs related to

174 flavonoid biosynthesis in this study. RT-PCR analysis was employed to confirm the results of the

175 RNA sequencing, and expressions of selected DEGs were measured in 3 different treatments.

176 Each sample replicated 3 times. Specific primers were designed by Primer Premier 5 software

177 (Table S1). Gene expression under different conditions was measured with the CFX96 ${ }^{\mathrm{TM}}$ Real-

178 time System (Bio-Rad, USA) using SYBR Premix Ex Taq II (TaKaRa, Japan). The 25S rRNA

179 gene obtained from Carthamus tinctorius L. was used as the internal reference gene to identify

180 differences in each cDNA template. The RT-PCR cycling conditions were as follows: $95^{\circ} \mathrm{C}$ for 3

$181 \mathrm{~min}$, followed by 40 cycles of $95^{\circ} \mathrm{C}$ for $10 \mathrm{~s}$ and $61^{\circ} \mathrm{C}$ for $30 \mathrm{~s}$. The $2^{-\Delta \Delta \mathrm{CT}}$ method (Livak \&

182 Schmittgen, 2001) was employed to analyze relative gene expressions. ANOVA with a post-hoc

183 Tukey-Test in SPSS (version 20) was used for data analysis. Three HCT genes, two FLS genes

184 and two ANS genes related to flavonoid biosynthesis, namely, CtHCT1, CtHCT2, CtHCT3,

$185 C t F L S 1, C t F L S 2, C t A N S 1$ and CtANS2, were successfully amplified and chosen to certify the

186 results.

187 Flavonoid quantification

188 Total flavonoid content was determined by the colorimetric method as previously described ( $Y u$ 189 et al., 2010) with some modifications. Powdered flower tissue $(0.2 \mathrm{~g})$ was dissolved in $10 \mathrm{ml}$ of

$19050 \%(\mathrm{v} / \mathrm{v})$ methanol and subjected to ultrasonic extraction for $90 \mathrm{~min}$. Scanning in the

191 wavelength range of 200 to $600 \mathrm{~nm}$, the optimum absorption peak wavelength was $255 \mathrm{~nm}$.

192 Therefore, the absorbance of the solution was measured at $255 \mathrm{~nm}$ by SpectraMax iD3

193 (Molecular, USA). Each sample replicated 3 times. ANOVA with a post-hoc Tukey-Test in

194 SPSS was used for data analysis.

195 To determine the HSYA content, $0.4 \mathrm{~g}$ of powdered flower tissue was dissolved in $50 \mathrm{ml}$ of $25 \%$

$196(\mathrm{v} / \mathrm{v})$ methanol and then subjected to ultrasonic extraction for $40 \mathrm{~min}$. The samples were then

197 diluted with methanol, filtered through a $0.45 \mu \mathrm{m}$ filter membrane and analyzed by HPLC

198 (Agilent 1200, USA). The HSYA content was analyzed with an Agilent C18 chromatographic

199 column $(4.6 \mathrm{~mm} \times 250 \mathrm{~mm}, 5 \mu \mathrm{m})$ in conjunction with a mobile phase that consisted of

200 methanol, acetonitrile and $0.7 \%(\mathrm{v} / \mathrm{v})$ phosphoric acid (26:2:72) for elution. The flow rate was

$2010.8 \mathrm{ml} \mathrm{min}^{-1}$, the injection volume was $10 \mu \mathrm{l}$, and the UV detector was set such that $\lambda=407 \mathrm{~nm}$.

202 To determine the kaempferol content, $1.0 \mathrm{~g}$ of powdered flower tissue was dissolved in $25 \mathrm{ml}$ of

203 methanol and then subjected to heat reflux extraction at $95^{\circ} \mathrm{C}$ for $30 \mathrm{~min}$ followed by

204 hydrochloric acid hydrolysis for $30 \mathrm{~min}$. The sample solutions were diluted with methanol,

205 filtered through a $0.45 \mu \mathrm{m}$ filter membrane and analyzed by HPLC. The kaempferol content was

206 analyzed with an Agilent C18 chromatographic column in conjunction with a mobile phase

207 consisting of methanol and $0.4 \%(\mathrm{v} / \mathrm{v})$ phosphoric acid for elution. The flow rate was $1.0 \mathrm{ml} \mathrm{min}^{-}$ 
208 , the injection volume was $20 \mu \mathrm{l}$, and the UV detector was set such that $\lambda=360 \mathrm{~nm}$ and $254 \mathrm{~nm}$.

209 Each sample replicated 3 times. ANOVA with a post-hoc Tukey-Test in SPSS was used for data 210 analysis.

\section{Results}

\section{Sequencing and functional annotation}

213 By the use of the BGISEQ-500 high-throughput sequencing platform, a total of $99.16 \mathrm{~Gb}$ of

214 clean data were obtained. The Q30 fraction of each sample was greater than or equal to $88.41 \%$.

215 Clean reads quality metrics were shown in Table S2. These results indicated that the quality of

216 the sequencing data was sufficient to continue with the following analysis. 129,544 Unigenes

217 were generated with a total length, mean length, N50 and GC content of 170,757,704 bp, 1,318

218 bp, 2,090 bp, and 41.59\%, respectively. The distribution of the Unigenes lengths was shown in

219 Figure 2. The Unigenes quality metrics were shown in Table 1.

220 Finally, a total of 78,179 Unigenes were annotated according to 7 functional databases. Among

221 all annotated Unigenes, 47,750 were annotated via the SwissProt database, 53,683 were

222 annotated via the KEGG database, and 23,382 were annotated by the GO database (Table 2).

223 Analysis of DEGs under different light intensities

224 Based on their gene expression levels, DEGs between groups were identified using the methods

225 described above. A total of 1,938 DEGs were identified among all Unigenes. Comparison of the

226 DEGs in the three different light intensity conditions revealed 1,249, 242, and 698 DEGs when

227 the following conditions were compared: HL vs LL, HL vs ML, and ML vs LL, respectively.

228 The distributions of the upregulated and downregulated Unigenes are shown in Figure 3.

229 According to GO functional analysis and significant enrichment analysis of the DEGs, three

230 comparisons showed a similar functional enrichment (Fig. 4). The "biological process" category

231 was the most enriched, followed by the "cellular component" category. In the biological process

232 category, the "metabolic processes", "cellular processes" and "single-organism processes" terms

233 were significantly enriched. In the cellular component category, "cell”, "cell part" and

234 "membrane" were the three largest categories. In the molecular function category, the two largest

235 categories were "binding" and "catalytic activity".

236 According to KEGG functional enrichment analysis, the "Metabolism" pathway was the most

237 enriched pathway in all three comparisons. A total of 110 relevant metabolic pathways were

238 identified by KEGG pathway analysis (Fig. 4).

239 To further investigate the Unigenes involved in flavonoid biosynthesis under different light

240 conditions, 13 DEGs related to flavonoid biosynthesis were screened and identified through GO

241 and KEGG (Table S3). Their FPKM (Fragments Per Kilobase of transcript per Million fragments

242 mapped) were showed in Figure 5 and sequence data were listed in supplemental files. 
243 RT-PCR analysis of DEGs involved in flavonoid biosynthesis

2447 of 13 DEGs related to flavonoid biosynthesis were successfully confirmed by RT-PCR.

245 CtHCT1 and CtHCT2 were significantly downregulated in the LL condition compared to their

246 expression in the other groups. However, CtHCT3 was downregulated in ML and upregulated in

247 LL. Regarding the FLS and ANS genes, CtFLS1 and CtANS1 were upregulated in LL, but

$248 C t F L S 2$ and CtANS2 were downregulated in LL compared to their expression in other treatment

249 groups (Fig. 6). These results indicated that a decrease in light intensity had a remarkable impact

250 on the expression of flavonoid biosynthesis related genes in safflower flowers, and the decrease

251 in light intensity to different degrees resulted in different effects on the expression of

252 homologous genes.

\section{Analysis of flavonoid content under different light intensities}

The total flavonoid content reached a maximum in the ML condition, followed by that in the HL condition, and the lowest flavonoid content was measured in the LL condition. However, the differences of total flavonoid contents among three conditions were not significant. The HSYA content showed little change between the ML and LL conditions but was significant decreased in the HL condition. However, kaempferol was present at a different concentration than HSYA; it was highest in the ML condition and was distinctly lower in both the HL and LL conditions (Fig. 7). These results indicated that a reduction in the light intensity within a suitable range was advantageous for flavonoid accumulation in safflower. In addition, the kaempferol concentration showed a significant negative relationship with CtHCT3 expression $(P<0.01)$, but no significant relationships with other DEGs. The authors suggest that flavonoid biosynthesis was affected by the differential expression of HCT genes, especially CtHCT3, in safflower flowers grown under different light intensities.

\section{Discussion}

The molecular mechanisms of flavonoid biosynthesis in safflower have not yet been defined. Huang (Huang et al., 2012) used Illumina-based de novo transcriptome sequencing to discover all known genes and primary metabolic pathways in this transcriptome. A total of 156 Unigenes that encode enzymes involved in flavonoid synthesis were identified based on the KEGG annotation. Liu (Liu et al., 2015) used 454 pyrosequencing to investigate genes related to the biosynthesis of safflor yellow, and 22 Unigenes involved in flavonoid biosynthesis were identified. In our previous research (Chen et al., 2018), by three-generation sequencing (PacBio RS II platform), 44 unique isoforms encoding enzymes involved in flavonoid biosynthesis were screened. However, 104 Unigenes were related with flavonoid biosynthesis in this experiment and 13 DEGs related with flavonoids biosynthesis were not found in all above studies. Due to different depths of sequencing, the deeper the depth of the sequencing is, the more functional 
278

279

280

281

282

283

284

285

286

287

288

289

290

291

292

293

294

295

296

297

298

299

300

301

302

303

304

305

306

307

308

309

310

311

312

313

genes that will be annotated. For instance, $99.16 \mathrm{~GB}$ of data were obtained in this experiment, but only $4.69 \mathrm{~Gb}$ of clean reads was obtained by Huang (Huang et al., 2012), and $10.43 \mathrm{~Gb}$ of clean data was obtained by three-generation sequencing. On the other hand, it was the first time that the relationship between light intensity and safflower flavonoid biosynthesis had been studied. Accordingly, 7 DEGs confirmed in this research had not been matched with previous researches.

In some species, increased light intensity can promote flavonoid accumulation (Deng et al., 2012; Wang et al., 2018). However, some species can increase the content of flavonoid under low light intensity to some extent ( $L$ u et al., 2017; Zhu, 2010). In this experiment, although safflower growth was better in high light intensity, flavonoid content reached the highest level under ML condition.

In Arabidopsis thaliana, HCT repression had a major impact on phenylpropanoid metabolism and resulted in the redirection of metabolic flux towards the flavonoid pathway (Besseau et al., 2007). In our previous study (Chen et al., 2018), 14 HCT genes were identified from safflower via full-length sequencing. Three of $14 H C T$ genes responded to MeJA treatment, and two of them were significantly regulated by MeJA. Accordingly, the contents of many flavonoids were significantly stimulated under MeJA treatment. It was indicated that $H C T$ genes were closely related to flavonoid biosynthesis in safflower.

In this experiment, $C t H C T 1$ and $C t H C T 2$ were downregulated under LL conditions, but CtHCT3 was upregulated under LL conditions. Accordingly, the HSYA content was the highest in the LL condition, but the total flavonoid and kaempferol concentrations were the lowest in the LL condition. In contrast, CtHCT3 was downregulated in the ML condition. Accordingly, the contents of total flavonoid, HSYA and kaempferol increased in the ML condition. In addition, the kaempferol content showed a significant negative relationship with $C t H C T 3$ expression. Therefore, we believe that the downregulation of $C t H C T 3$ may have a positive effect on flavonoid accumulation. On the other hand, phylogenetic relationship among $3 H C T$ genes showed that $C t H C T 1$ and $C t H C T 2$ had closer genetic relationship with each other than CtHCT3 (Fig. S1). Thus, we suggested that $3 H C T$ genes in safflower had different subfunction: $C t H C T 1$ and CtHCT2 may had protective function under high light intensity, and CtHCT3 might be closely related to flavonoid biosynthesis.

However, the different trends observed for HSYA and kaempferol accumulation might be attributed to their different synthesis pathways and effects from other genes such as $F L S$ and $A N S$. The expression levels of three $H C T$, two $F L S$ and two $A N S$ genes in safflower flowers grown under different light intensities were analysed, but which homologous gene played a major role in flavonoid biosynthesis requires further study.

Through the above experiments, we suggested that middle light intensity could promotes 
314 flavonoids accumulation in safflower. We hypothesized that a decrease in light intensity led to

315 the downregulation of HCT genes in safflower, then the downregulation of HCT genes might

316 result in the repression of lignin synthesis and increased substrate catalysis by CHS, promoting

317 flavonoid accumulation. It was also found that the growth of safflower plants was inhibited when

318 the light intensity was reduced, which might have affected HCT expression and the repression of

319 lignin synthesis. Therefore, the authors believe that safflower grew vigorously under high light

320 conditions, but a reduction in the light intensity within a suitable range promoted flavonoid

321 biosynthesis in safflower flowers.

\section{Conclusions}

323 This is the first transcriptome analysis of Carthamus tinctorius L. exposed to different light conditions. We obtained a total of $99.16 \mathrm{~Gb}$ of clean reads. A total of 129,544 Unigenes were identified, 78,179 (60.3\%) of which were functionally annotated. A considerable number of high-quality Unigenes were identified that will be useful for future studies. 13 novel genes related to flavonoid biosynthesis were identified based on the functional annotation of differentially expressed genes, and the differential expressions of 7 of them under different light intensities were analyzed by RT-PCR. The concentrations of total flavonoid and two active compounds were measured in safflower flowers grown under 3 different light conditions. We confirmed that the expression of HCT genes played an important role in flavonoid biosynthesis in safflower flowers grown under different light intensities. Our study lays a foundation for further research on the effects of light on flavonoid biosynthesis in safflower flowers.

\section{Acknowledgements}

Many thanks go to the participating families and the entire study team.

\section{References}

Asgarpanah J, Kazemivash N. 2013. Phytochemistry, pharmacology and medicinal properties of Carthamus tinctorius L. Chinese Journal of Integrative Medicine 19(2):153-159 DOI 10.1007/s11655-013-1354-5.

Ashri A, Knowles P, Urie A, Zimmer D, Cahaner A, Marani A. 1977. Evaluation of the germplasm collection of safflower Carthamus tinctorius III Oil content and iodine value and their associations with other characters. Economic Botany 31(1):38-46 DOI 10.1007/BF02860650.

Besseau S, Hoffmann L, Geoffroy P, Lapierre C, Pollet B, Legrand M. 2007. Flavonoid accumulation in arabidopsis repressed in lignin synthesis affects auxin transport and plant growth. Plant Cell 19(1):148-162 DOI 10.1105/tpc.106.044495. 
Chen J, Tang X, Ren C, Wei B, Wu Y, Wu Q, Pei J. 2018. Full-length transcriptome sequences and the identification of putative genes for flavonoid biosynthesis in safflower. BMC Genomics 19(1):548 DOI 10.1186/s12864-018-4946-9.

Chinese Pharmacopoeia Commission. 2015. Pharmacopoeia of the People's Republic of China. Beijing: China Medical Science Press.

Dempewolf H, Rieseberg L, Cronk Q. 2008. Crop domestication in the Compositae: a familywide trait assessment. Genetic Resources and Crop Evolution 55(8):1141-1157 DOI 10.1007/s10722-008-9315-0.

Deng B, Shang X, Fang S, Li Q, Fu X, Su J. 2012. Integrated effects of light intensity and fertilization on growth and flavonoid accumulation in Cyclocarya paliurus. Journal of Agricultural and Food Chemistry 60(25):6286-6292 DOI 10.1021/jf301525s.

Devi K, Malar D, Nabavi S, Sureda A, Xiao J, Nabavi S, Daglia M. 2015. Kaempferol and inflammation: From chemistry to medicine. Pharmacological Research 99:1-10 DOI 10.1016/j.phrs.2015.05.002.

Du H, Huang Y, Tang Y. 2010. Genetic and metabolic engineering of isoflavonoid biosynthesis. Applied Microbiology and Biotechnology 86(5):1293-1312 DOI 10.1007/s00253-010-2512-8.

Gao S, Chang C, Niu X, Li L, Zhang Y, Gao J. 2018. Topical application of hydroxysafflor yellow A accelerates the wound healing in streptozotocin induced T1DM rats. European Journal of Pharmacology 823:72-78 DOI 10.1016/j.ejphar.2018.01.018.

Grabherr M, Haas B, Yassour M, Levin J, Thompson D, Amit I, Adiconis X, Fan L, Raychowdhury R, Zeng Q, Chen Z, Mauceli E, Hacohen N, Gnirke A, Rhind N, di Palma F, Birren B, Nusbaum C, Lindblad-Toh K, Friedman N, Regev A. 2011. Fulllength transcriptome assembly from RNA-Seq data without a reference genome. Nature Biotechnology 29(7):644-652 DOI 10.1038/nbt.1883.

Guan LL, Wu W, Zheng YL, Wang T. 2007. Correlation analysis between photosynthesis and yield of the leaves at different positions of safflower. Acta Agronomica Sinica 33(8):13521359 DOI 10.3321/j.issn:0496-3490.2007.08.022.

Guo D, Gao Y, Liu F, He B, Jia X, Meng F, Zhang H, Guo M. 2019. Integrating molecular characterization and metabolites profile revealed CtCHI1's significant role in Carthamus tinctorius L. BMC Plant Biology 19(1):376 DOI 10.1186/s12870-019-1962-0.

Hai D, Huang Y, Tang Y. 2010. Genetic and metabolic engineering of isoflavonoid biosynthesis. Applied Microbiology and Biotechnology 86(5):1293-1312 DOI 10.1007/s00253-010-2512-8.

Hong B, Wang Z, Xu T, Li C, Li W. 2015. Matrix solid-phase dispersion extraction followed by high performance liquid chromatography-diode array detection and ultraperformance 
liquid chromatography-quadrupole-time of flight-mass spectrometer method for the determination of the main compounds from Carthamus tinctorius L. (Hong-hua). Journal of Pharmaceutical and Biomedical Analysis 107:464-472 DOI 10.1016/j.jpba.2015.01.040.

Huang L, Yang X, Sun P, Tong W, Hu S. 2012. The first illumina-based de novo transcriptome sequencing and analysis of safflower flowers. PLoS One 7(6):e38653 DOI 10.1371/journal.pone.0038653.

Jiao Y, Lau OS, Deng XW. 2007. Light-regulated transcriptional networks in higher plants. Nature Reviews Genetics 8(3):217-230 DOI 10.1038/nrg2049.

Jin Y, Xiao Y, Zhang F, Xue X, Xu Q, Liang X. 2008. Systematic screening and characterization of flavonoid glycosides in Carthamus tinctorius L. by liquid chromatography/UV diode-array detection/electrospray ionization tandem mass spectrometry. Journal of Pharmaceutical and Biomedical Analysis 46(3):418-430 DOI 10.1016/j.jpba.2007.10.036.

Knowles P. 1969. Centers of plant diversity and conservation of crop germplasm: safflower. Economic Botany 23(4):324-329 DOI 10.2307/4253083.

Knowles P. 1972. The plant geneticist's contribution toward changing lipid and amino acid composition of safflower. Journal of the American Oil Chemists Society 49(1):30-32 DOI 10.1007/BF02545134.

Langmead B, Salzberg SL. 2012. Fast gapped-read alignment with Bowtie 2. Nature Methods. 9:357-359 DOI 10.1038/nmeth.1923.

Li B, Dewey CN. 2011. RSEM: accurate transcript quantification from RNA-Seq data with or without a reference genome. BMC Bioinformatics 12:323 DOI 10.1186/1471-2105-12-323.

Liu X, Dong Y, Yao N, Zhang Y, Wang N, Cui X, Li X, Wang Y, Wang F, Yang J, Guan L, Du L, Li H, Li X. 2015. De novo sequencing and analysis of the safflower transcriptome to discover putative genes associated with safflor yellow in Carthamus tinctorius $\mathrm{L}$. International Journal of Molecular Sciences 16(10):25657-25677 DOI 10.3390/ijms 161025657.

Livak K, Schmittgen T. 2001. Analysis of relative gene expression data using real-time quantitative PCR and the $2^{-\Delta \Delta C T}$ method. Methods 25(4):402-408 DOI 10.1006/meth.2001.1262.

Love MI, Huber W, Anders S. 2014. Moderated estimation of fold change and dispersion for RNA-seq data with DESeq2. Genome Biology 15(12):550 DOI 10.1186/s13059-014-05508.

Lu Y, Bu Y, Hao S, Wang Y, Zhang J, Tian J, Yao Y. 2017. MYBs affect the variation in the ratio of anthocyanin and flavanol in fruit peel and flesh in response to shade. Journal of Photochemistry and Photobiology B: Biology 168:40-49 DOI 
10.1016/j.jphotobiol.2017.01.017.

Pan J, Chen H, Guo B, Liu C. 2017. Understanding the molecular mechanisms underlying the effects of light intensity on flavonoid production by RNA-seq analysis in Epimedium pseudowushanense B.L.Guo. PLoS ONE 12(8):e0182348 DOI 10.1371/journal.pone.0182348.

Pertea G, Huang X, Liang F, Antonescu V, Sultana R, Karamycheva S, Lee Y, White J, Cheung F, Parvizi B, Tsai J, Quackenbush J. 2003. TIGR Gene Indices clustering tools (TGICL): a software system for fast clustering of large EST datasets. Bioinformatics 19(5):651-652 DOI 10.1093/bioinformatics/btg034.

Ren C, Tang X, Chen C, Chen J, Pei J, Wu Y, Wu Q. 2019. Cloning and expression analysis of a new chalcone isomerase gene during flowering in safflower. Turkish Journal of Botany 43(2):143-150 DOI 10.3906/bot-1809-25.

Saito K, Yonekura-Sakakibara K, Nakabayashi R, Higashi Y, Yamazaki M, Tohge T, Fernie AR. 2013. The flavonoid biosynthetic pathway in Arabidopsis: structural and genetic diversity. Plant Physiology and Biochemistry 72:21-34 DOI 10.1016/j.plaphy.2013.02.001.

Tian L, Pang Y, Dixon R. 2008. Biosynthesis and genetic engineering of proanthocyanidins and (iso) flavonoid. Phytochemistry Reviews 7(3):445-465 DOI 10.1007/s11101-007-9076-y.

Tu YH, Liu F, Guo DD, Fan L, Zhu Z, Xue Y, Gao Y, Guo M. 2016. Molecular characterization of flavanone 3-hydroxylase gene and flavonoid accumulation in two chemotyped safflower lines in response to methyl jasmonate stimulation. BMC Plant Biology 16(1):132 DOI 10.1186/s12870-016-0813-5.

Wang J, Guo M, Li Y, Wu R, Zhang K. 2018. High-throughput transcriptome sequencing reveals the role of anthocyanin metabolism in Begonia semperflorens under high light stress. Photochemistry and Photobiology 94(1):105-114 DOI 10.1111/php.12813.

Wu YY. 2017. The main environmental factors on quality and mechanism study in Carthamus tinctorius L. M.S. Thesis, Chengdu University of Traditional Chinese Medicine.

Yu D, Cheng YS, Hu SQ, Zhang H. 2010. Determination of total flavonoids and kaempferol-3O-rutinoside in safflower produced in Sichuan and safflower variety named white flower. West China Journal of Pharmaceutical Sciences 25(6):751-753 DOI 10.13375/j.cnki.wcjps.2010.06.006.

Zhou D, Qu Z, Wang H, Su Y, Wang Y, Zhang W, Wang Z, Xu Q. 2018. The effect of hydroxysafflower yellow A on coronary heart disease through Bcl-2/Bax and PPAR- $\gamma$. Experimental and Therapeutic Medicine 15(1):520-526 DOI 10.3892/etm.2017.5414.

Zhu CC. 2010. Effects of environmental factors on the growth and principal medicinal compositions of Ginkgo biloba seedlings. Ph.D. Thesis, Nanjing Forestry University. 
455 Zou J, Wang N, Liu M, Bai Y, Wang H, Liu K, Zhang H, Xiao X, Wang K. 2018. Nucleolin 456 mediated pro-angiogenic role of hydroxysafflor yellow A in ischaemic cardiac dysfunction: 457 post-transcriptional regulation of VEGF-A and MMP-9. Journal of Cellular and Molecular $458 \quad$ Medicine 22(5):2692-2705 DOI 10.1111/jcmm.13552. 


\section{Table $\mathbf{1}$ (on next page)}

\section{Quality metrics of Unigenes}

N50: The N50 length is used to determine the assembly continuity, the higher the better. N70: Similar to N50. N90: Similar to N50. GC (\%): the percentage of $\mathrm{G}$ and C bases in all Unigenes 


\begin{tabular}{llllllll}
\hline Sample & $\begin{array}{l}\text { Total } \\
\text { Number }\end{array}$ & $\begin{array}{l}\text { Total } \\
\text { Length }\end{array}$ & $\begin{array}{l}\text { Mean } \\
\text { Length }\end{array}$ & N50 & N70 & N90 & GC (\%) \\
\hline HL_1 & 47956 & 48017129 & 1001 & 1597 & 1038 & 422 & 43.49 \\
HL_2 & 42932 & 40607825 & 945 & 1520 & 961 & 388 & 43.43 \\
HL_3 & 42861 & 40604125 & 947 & 1514 & 959 & 391 & 43.6 \\
LL_1 & 72845 & 71641113 & 983 & 1631 & 1007 & 391 & 41.59 \\
LL_2 & 59271 & 56435088 & 952 & 1589 & 987 & 376 & 42.65 \\
LL_3 & 61471 & 58975317 & 959 & 1594 & 998 & 380 & 42.47 \\
ML_1 & 51245 & 51521358 & 1005 & 1626 & 1054 & 417 & 43.03 \\
ML_2 & 48597 & 49086125 & 1010 & 1619 & 1059 & 421 & 43.33 \\
ML_3 & 60239 & 53701642 & 891 & 1510 & 896 & 344 & 42.54 \\
\hline
\end{tabular}


Table 2 (on next page)

Unigene annotation and statistics for safflower de novo transcriptome . 


\begin{tabular}{lll}
\hline Categories & Number & Frequency \\
\hline Total & 129544 & $100 \%$ \\
NR & 70977 & $54.79 \%$ \\
NT & 45716 & $35.29 \%$ \\
SwissProt & 47750 & $36.86 \%$ \\
KEGG & 53683 & $41.44 \%$ \\
KOG & 58385 & $45.07 \%$ \\
Interpro & 61507 & $47.48 \%$ \\
GO & 23382 & $18.05 \%$ \\
All annotated Unigenes & 78179 & $60.35 \%$ \\
\hline
\end{tabular}

1 


\section{Figure 1}

Proposed a part of flavonoid biosynthesis pathway in safflower.

$\mathrm{HCT}=$ Shikimate O-hydroxycinnamoyl transferase; $\mathrm{CHS}=$ Chalcone synthase; FLS = Flavonol synthase; ANS = Anthocyanidin synthase; HSYA = Hydroxysafflor yellow A.

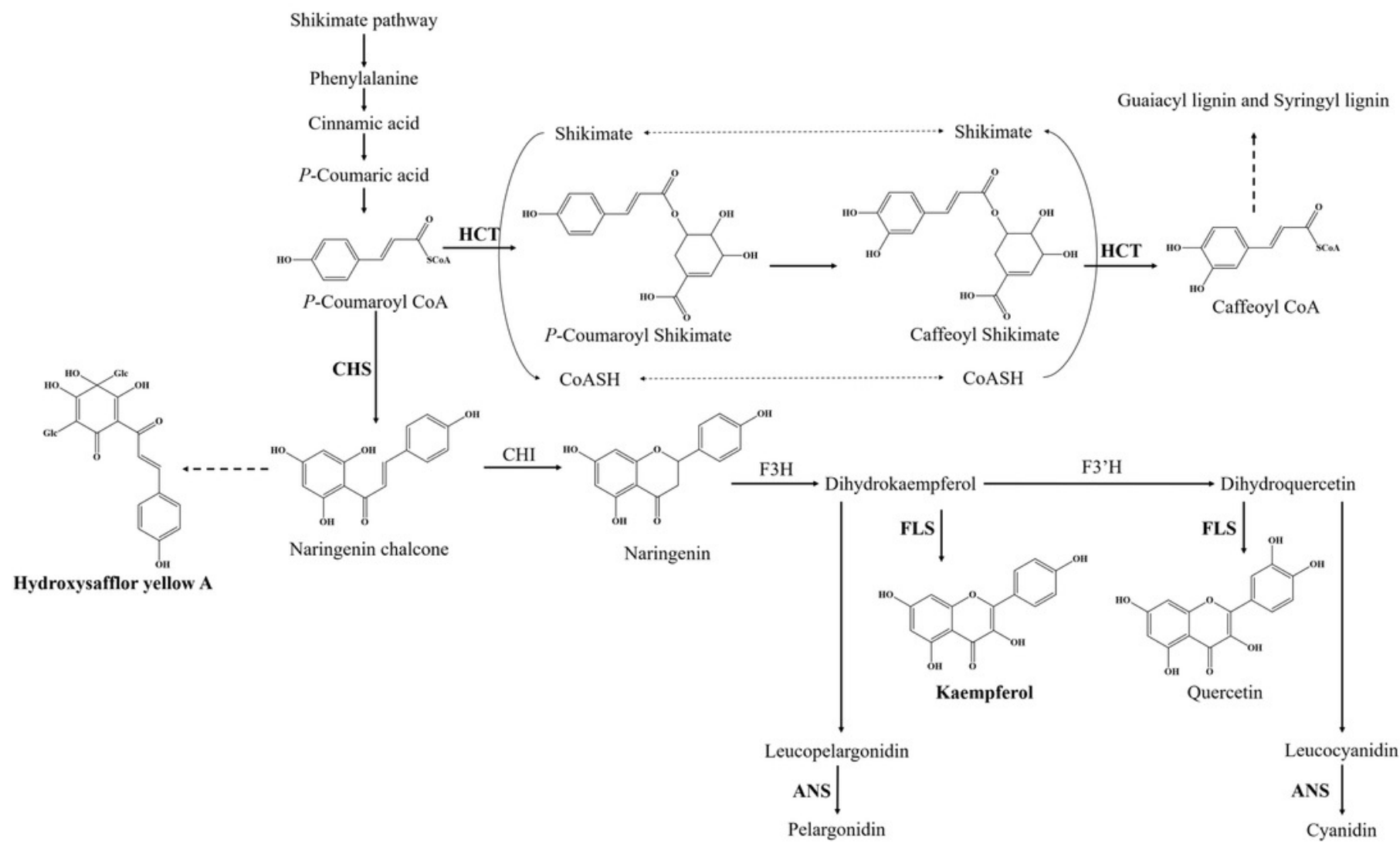


Figure 2

Length distribution of All-Unigene in safflower transcriptome.

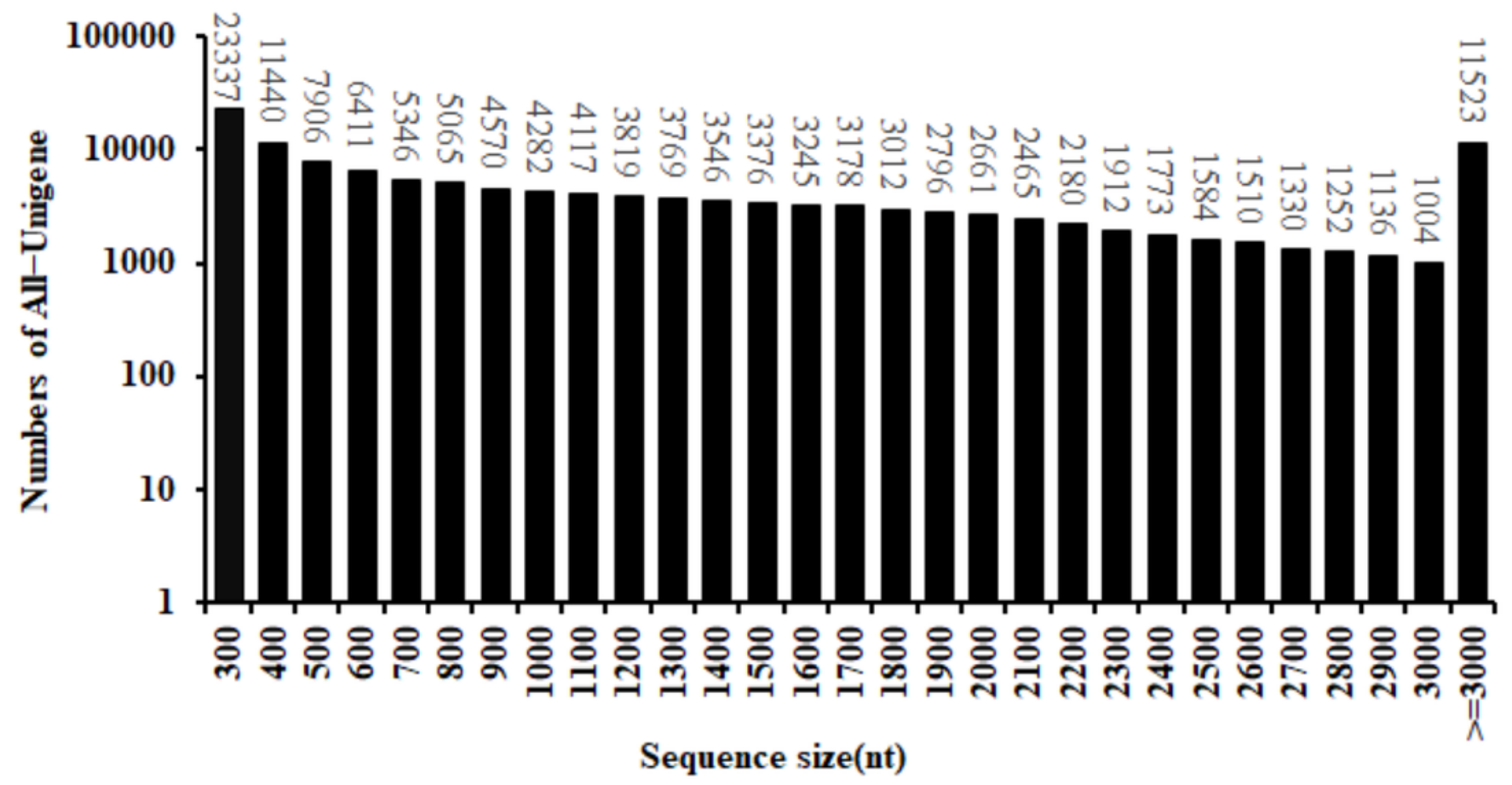




\section{Figure 3}

Statistic of differently expressed genes obtained by DEseq2.

Red color represents up regulated DEGs. Blue color represents down regulated DEGs. HL = High light; ML = Middle light; LL = Low light.

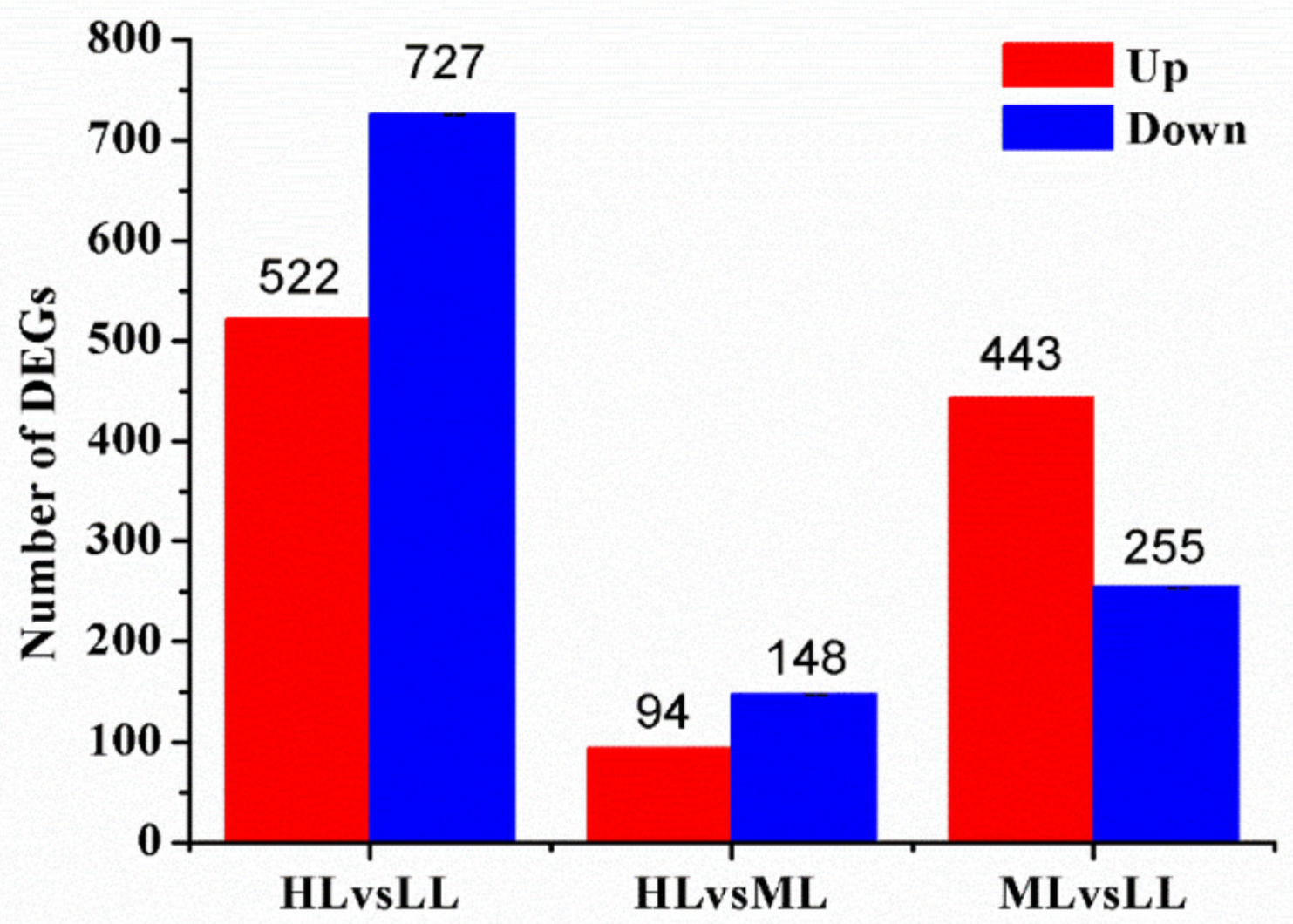


Figure 4

GO and KEGG pathway classifications of DEGs.

$X$ axis represents number of DEG. $Y$ axis represents $G O$ term in $A, B$ and $C$ : (A) Comparison of HLvsLL, (B) Comparison of HLvsML, (C) Comparison of MLvsLL; $Y$ axis represents functional classification of KEGG in D, E and F: (D) Comparison of HLvsLL, (E) Comparison of HLvsML, (F) Comparison of MLvsLL. 


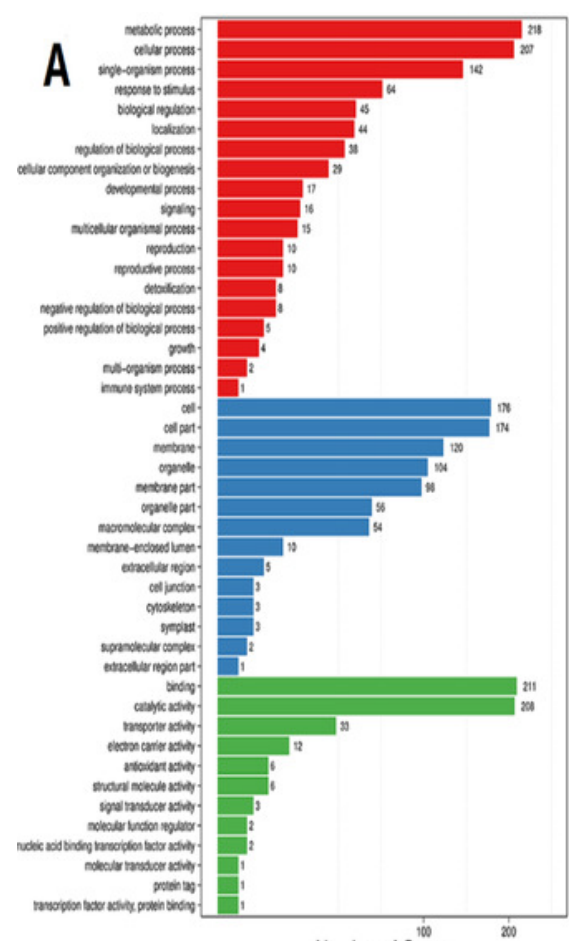

B
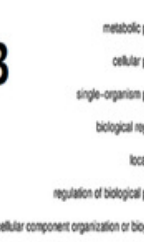

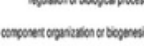

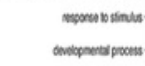

matcentur oprions pocons

acoposon pocent$$
\text { (1) }
$$$$
\text { nonom }
$$

$$
\text { monosour }
$$
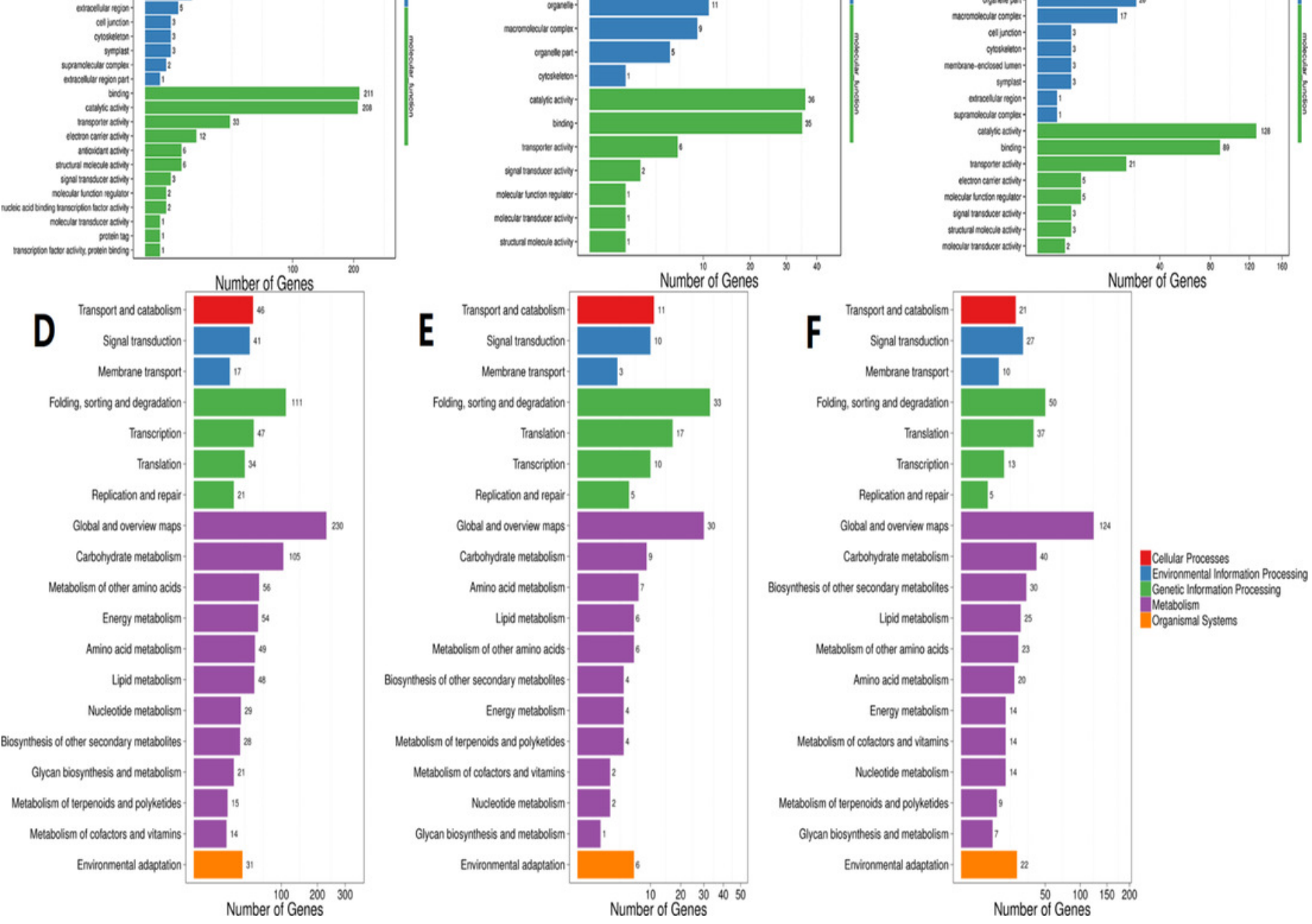
Figure 5

Expression levels of the DEGs related to flavonoid biosynthesis.

13 selected differential expressed genes related to flavonoid biosynthesis from the transcriptome and average FKPM values for biological replicates are shown. (A)-(G) presented CtHCT1, CtHCT2, CtHCT3, CtFLS1, CtFLS2, CtANS1 and CtANS2 respectively. 

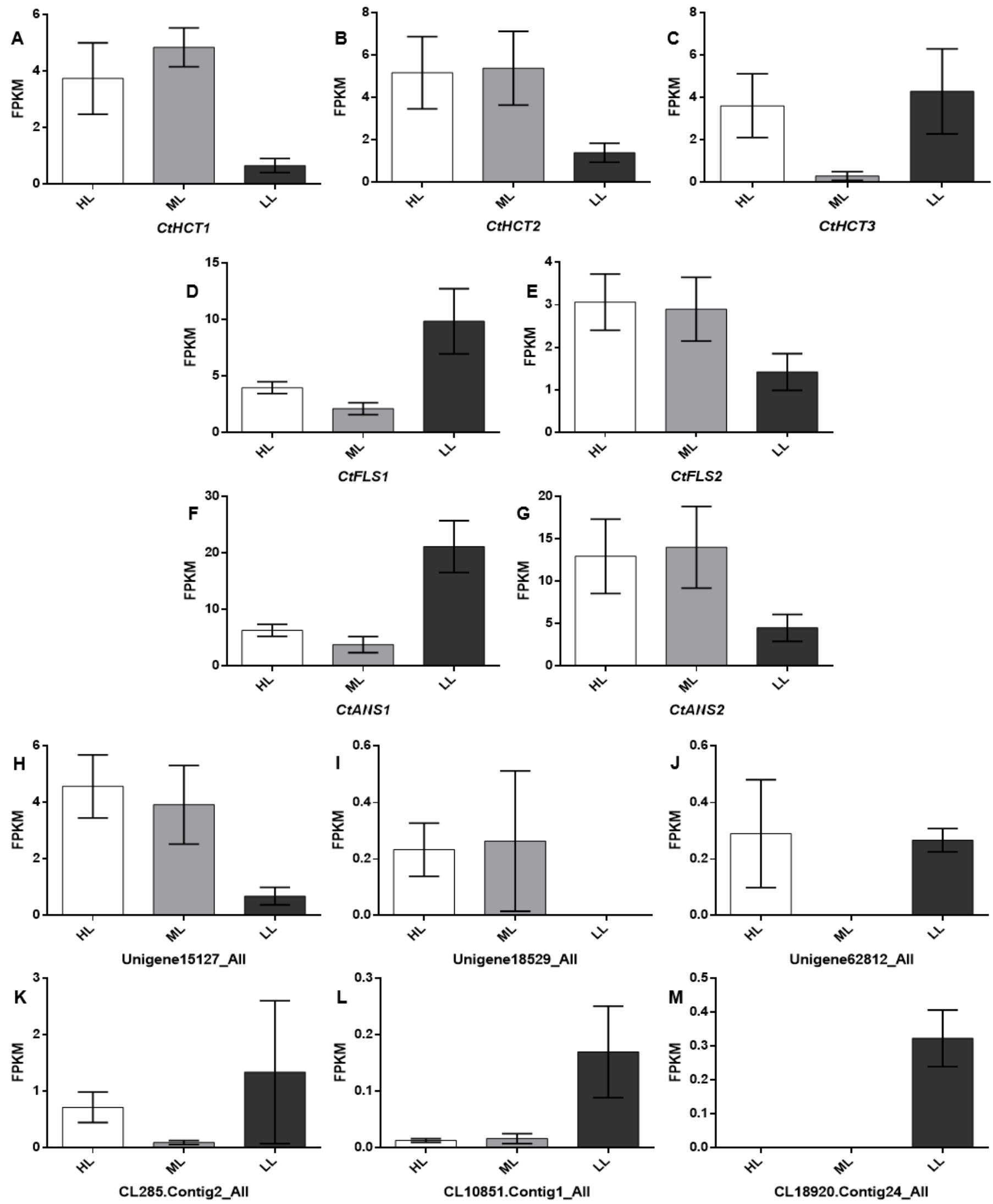
Figure 6

Expression analysis by RT-PCR of seven flavonoid related genes under different light intensities.

(A)-(G) presented CtHCT1, CtHCT2, CtHCT3, CtFLS1, CtFLS2, CtANS1 and CtANS2 respectively. Results of ANOVA with a post-hoc Tukey- Test are shown with asterisks $(* P<0.05, * * P<0.01)$.
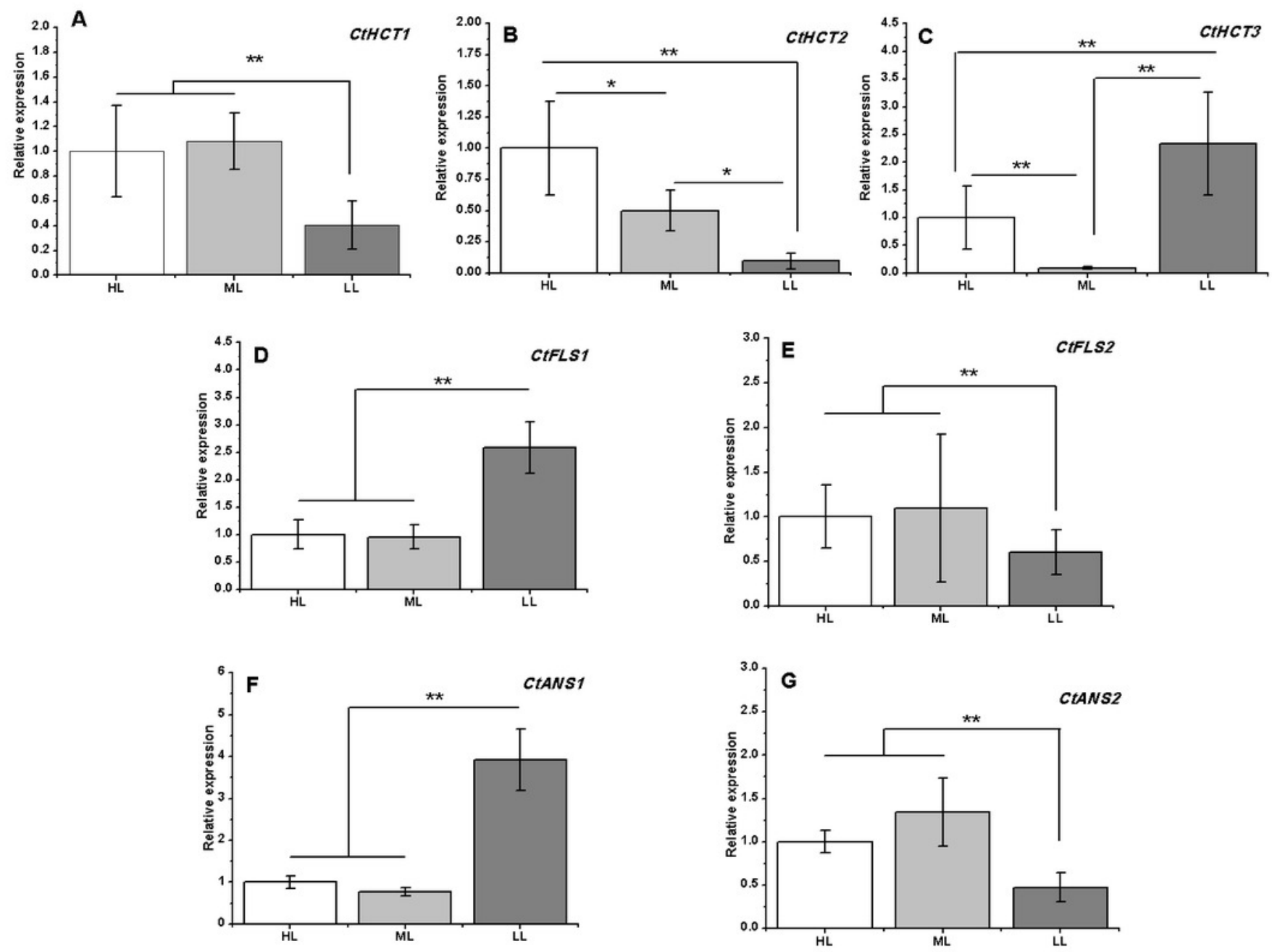
Figure 7

The flavonoids concentration in safflower flowers under different light intensities.

(A)-(C) presented contents of total flavonoids, hydroxysafflor yellow A (HSYA) and kaempferol respectively. Results of ANOVA with a post-hoc Tukey- Test are shown with asterisks $\left(* P<0.05,{ }^{* * P}<0.01\right)$.
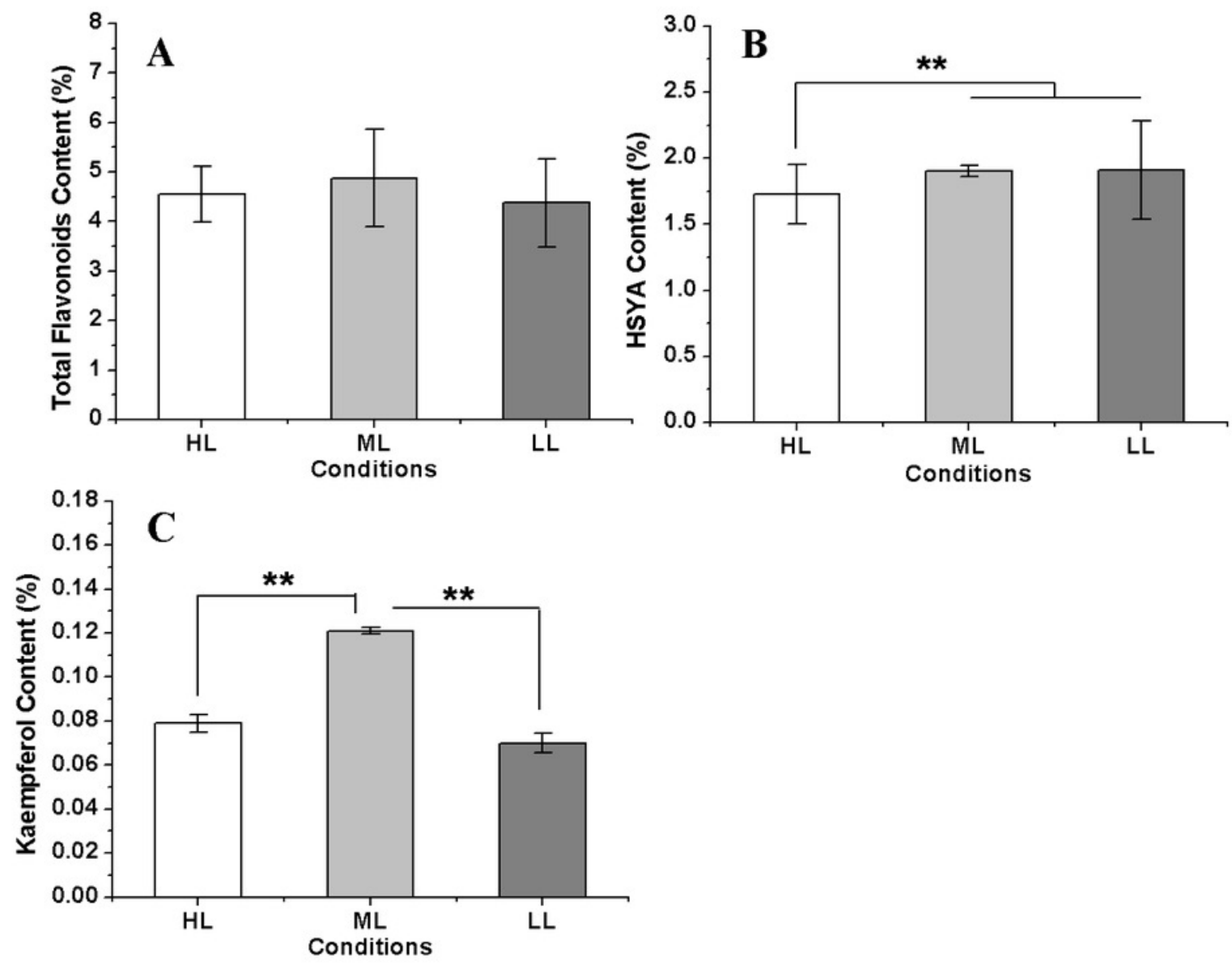